\title{
RENDIMIENTO Y ESTABILIDAD DE HÍBRIDOS DE MAÍZ DE CRUZAS SIMPLES EVALUADAS EN COLOMBIA, MÉXICO Y CENTRO AMÉRICA
}

\author{
G. Srinivasan*, S.K. Vasal*, F. González Ceniceros*, H. Córdova*, S. Pandey** y \\ N. Vergara*
}

\begin{abstract}
RESUMEN
Tres ensayos de híbridos (SC9007,SC9008, SC9009) fueron evaluados en siete u ocho localidades en México, Centro y Sudamérica durante 1990-91. 68 híbridos blancos se incluyeron en los ensayos SC9007 y SC9008 y 25 híbridos amarillos en el ensayo SC9009, junto con dos testigos locales en cada ensayo. Los híbridos más estables fueron: SCll, SC12, SC57 y SC44 entre los de grano blanco y el SC67 entre los de grano amarillo. SC07 (6.49 t/ha) y SC57 (5.95 t/ha) fueron los híbridos blancos con mayor rendimiento en los ensayos SC9007 y SC9008 respectivamente. Entre los híbridos amarillos, SC82 (5.94 t/ha), SC80 (5.93 t/ha) y SC77 (5.88 t/ha) fueron los de mejor comportamiento. Las ganancias máximas sobre los testigos se observaron en Cuyuta, Guatemala y EEEJN, Costa Rica. En Honduras, sin embargo, los mejores híbridos estuvieron a la par con el mejor testigo híbrido. En Panamá, los híbridos amarillos, scn, SC80 y SC82 son prometedores, mientras que en Colombia, los híbridos blancos SC03, SC11 y SC57 fueron los de mejor adaptación.
\end{abstract}

\begin{abstract}
Yield and Stability of Single Cross Maize Hybrids tested in Colombia, Mexico and Central America. Three hybrid trials (SC 9007, SC 9008, SC 9009) were evaluated in seven to eight locations in Mexico, Central and South America during 1990-91. Sixty-eight white hybrids were tested in SC 9007 and SC 9008, and 25 yellow hybrids in SC 9009 along with two local checks in each trial. SCl1, SC12, SC57, and SC44 among whites, and SC67 among yellows were the most stable hybrids. SC07 (6.49 t/ha.), and SC57 (5.95 t/ha) were the highest yielding white hybrids from SC 9007, and SC9008 respectively. Among yellows, SC82 (5.94 t/ha.), SC80 (5.93 $\mathrm{t} / \mathrm{ha}$.), and $\operatorname{sen}(5.88 \mathrm{t} / \mathrm{ha}$.) were the best performers. Maximum gains over the checks were observed in Cuyuta, Guatemala, and EEEJn, Costa Rica. In Honduras, however, the best hybrid was on par with the best local check hybrid. For Panama, the yellow hybrids, SC77, SC80, SC82 are promising. In Colombia, SC03, SCll, and SC57 among whites, were best adapted.
\end{abstract}

\section{INTRODUCCIÓN}

El programa de híbridos de maíz de CIMMYT se inició en 1985 en respuesta a la gran demanda de productos orientados a híbridos en muchos programas nacionales de países en desarrollo. En un período de seis años, el programa de híbridos de maíz de CIMMYT ha desarrollado una variedad amplia de fuentes de germoplasma orientado al desarrollo de híbridos (7). Se obtuvo información sobre patrones heteróticos y habilidad combinatoria de las poblaciones y pooles de CIMMYT $(1,2,4,8)$. Durante 1991, más de 100 líneas tropicales y subtropicales se desarrollaron en el programa de híbridos y fueron anunciadas para su distribución y es proporcionada a nuestros cooperadores que las soliciten.

La meta principal de cualquier programa de híbridos, es producir híbridos de cruzas simples involucrando líneas homocigotas como progenitores, con el objeto de explotar al máximo la heterosis y obtener híbridos más uniformes y atractivos. Sinembargo, durante el proceso para lograr esta meta, que podría llevar mucho tiempo y esfuerzo, uno debería de explotar las posibilidades de desarrollar tipos de híbridos convencionales modificados que incluyan dos padres, los cuales son relativamente fácil de producir en poco tiempo y tienen ciertas ventajas

* CIMMYT, Lisboa 27, Apdo. Postal 6-641, México, D.F., México.
** CIMMYT, c/o ClAT, Apdo. Aéreo 67-13, Cali, Colombia. 
$(5,6)$. Los objetivos de este estudio son: (i) identificar tipos superiores de híbridos simples modificados en el germoplasma tropical tardío blanco y amarillo, desarrollados en CIMMYT y (ii) determinar la estabilidad de rendimiento de esos híbridos a través de varios ambientes en Centro y Sudamérica.

\section{MATERIALES Y MÉTODOS}

Los híbridos simples modificados evaluados en este estudio incluyen como progenitores una línea endocriada y una línea reconstituída de generación temprana. Las líneas reconstituídas de generaciones tempranas fueron formadas recombinando líneas hermanas elite en S4 a S6, que se derivaron de un origen común a nivel de S2 o S3. Se evaluaron 68 híbridos blancos y 25 híbridos amarillos en tres ensayos que son: SC 9007, SC 9008 y SC 9009, en 7-8 localidades cada uno con 3 repeticiones (Cuadro 1). Los ensayos también incluyeron dos testigos locales. El diseño experimental usado fué el de bloques completos al azar para el ensayo SC9007 y de látice simple para los ensayos SC 9008 y SC 9009. La unidad experimental consistió de dos surcos de 5 metros espaciados a 75 - 90 $\mathrm{cm}$., con un espacio de $50 \mathrm{~cm}$ entre plantas. Se sembraron 3 semillas/golpe, para después aclarear a dos, resultando una densidad de plantas de 45,000 a 53,000 plantas por hectárea. Los dos ensayos de híbridos blancos, SC 9007 y SC9008, se evaluaron en siete localidades y el ensayo de híbridos amarillos (SC 9009) se evaluó en ocho localidades durante 1990-91. Se registraron datos de altura de planta y días a floración femenina en todas las localidades, excepto en Poza Rica, México; donde los datos no pudieron ser colectados debido al daño causado por un huracán al tiempo de floración. El peso fresco de mazorca y la humedad de grano se registraron en la cosecha. El rendimiento de grano se calculó al $80 \%$ de desgrane y ajustanto al $15.5 \%$ de humedad. El análisis de varianza se llevó a cabo para rendimiento de grano y se estimaron los parámetros de estabilidad de Eberhart y Russell (3).

Cuadro 1. Características de los tres ensayos experimentales de cruzas simples.

\begin{tabular}{lcccc}
\hline $\begin{array}{c}\text { Ensayo } \\
\text { número }\end{array}$ & $\begin{array}{c}\text { No. de } \\
\text { entradas* }\end{array}$ & $\begin{array}{c}\text { Código de } \\
\text { Híbridos }\end{array}$ & $\begin{array}{c}\text { No. de } \\
\text { Localid. }\end{array}$ & $\begin{array}{r}\text { Adaptación, } \\
\text { Color de grano }\end{array}$ \\
\hline SC 9007 & 28 & SC01-SC26 & 7 & Tropical blanco \\
SC 9008 & 36 & SC27-SC60 & 7 & Tropical blanco \\
SC 9009 & 25 & SC61-SC83 & 8 & Tropical amariilo
\end{tabular}

* Incluidos dos testigos locales.

\section{RESULTADOS Y DISCUSIÓN}

Los promedios de rendimiento de grano, altura de planta, floración femenina y porciento de humedad de los tres ensayos en sus respectivas localidades, se indican en el Cuadro 2. Omonita, Honduras registró el promedio de rendimiento más alto (7.22 t/ha) en el ensayo SC 9007 y tuvo la altura de planta más corta. En el ensayo SC 9008, Palmira, Colombia; fué la localidad con el rendimiento más alto $(5.88$ t/ha). La misma localidad registró el más alto rendimiento en el ensayo de híbridos amarillos SC9009 y mostró también las plantas más altas y tardías. El análisis de varianza a través de localidades mostró diferencias altamente significativas para localidades, entradas y localidades x entradas (datos no mostrados).

Cuadro 2. Localidades y sus medias de rendimiento, altura de planta, floracion feminina y porcentaje de humedad para SC 9007, SC 9008, y SC 9009.

\begin{tabular}{|c|c|c|c|c|}
\hline $\begin{array}{l}\text { Localidad y país } \\
\text { y ensayo número }\end{array}$ & $\begin{array}{l}\text { Rendim. } \\
\text { grano } \\
\text { (t/ha) }\end{array}$ & $\begin{array}{c}\text { Altura } \\
\text { Planta } \\
(\mathrm{cm})\end{array}$ & $\begin{array}{l}\text { Días de } \\
\text { florac. }\end{array}$ & $\underset{\%}{\text { Hum. }}$ \\
\hline \multicolumn{5}{|l|}{ SC 9007} \\
\hline Cuyuta, Guatemala & 5,14 & 199 & 57,4 & 24,0 \\
\hline San Andrés, EL Salvador & or 4,34 & 207 & 59,0 & 30,5 \\
\hline Omonita, Honduras & 7,22 & 188 & 53,2 & 24,2 \\
\hline EEEJN, Costa Rica & 3,95 & 224 & 53,1 & 17,4 \\
\hline Palmira, Colombia & 5,06 & 233 & 61,0 & 18,4 \\
\hline Turipana, Colombia & 5,74 & 249 & 49,3 & 20,3 \\
\hline Poza Rica, México & 5,74 & - & - & 25,2 \\
\hline \multicolumn{5}{|l|}{ SC 9008} \\
\hline Cuyuta, Guatemala & 5,16 & 198 & 55,5 & 23,6 \\
\hline Las Vegas, Guatemala & 3,53 & 209 & 56,6 & 21,1 \\
\hline San Andrés, El Salvador & r 3,98 & 206 & 57,6 & 32,9 \\
\hline EEEJN, Costa Rica & 4,24 & 229 & 52,7 & 16,9 \\
\hline Palmira, Colombia & 5,88 & 246 & 59,2 & 18,3 \\
\hline Turipana, Colombia & 5,06 & 248 & 50,5 & 21,2 \\
\hline Poza Rica, México & 4,97 & - & - & 24,9 \\
\hline \multicolumn{5}{|l|}{ sc 9009} \\
\hline Cuyuta, Guatemala & 4,68 & 209 & 57,4 & 22,9 \\
\hline Santa Cruz, El Salvador & $r 4,43$ & 220 & 53,3 & 25,1 \\
\hline Las Acacias, Honduras & 4,29 & 232 & 56,8 & 19,0 \\
\hline La Honda, Panamá & 5,84 & 249 & 56,5 & 16,1 \\
\hline Parita, Panamá & 5,67 & 253 & 55,4 & 18,3 \\
\hline Palmira, Colombia & 6,16 & 256 & 63,9 & 19,0 \\
\hline Turipana, Colombia & 4,61 & 246 & 51,2 & 21,9 \\
\hline Poza Rica, México & 4,96 & - & - & 25,4 \\
\hline
\end{tabular}

Las diez cruzas con rendimientos superiores en cada uno de los tres ensayos aparecen en el Cuadro 3, con sus respectivos parámetros de estabilidad. En el ensayo SC 9007, las dos líneas derivadas de Pob. 21 HC 109 y Pob. 
$21 \mathrm{HC} 219$, aparecen como progenitores en cuatro de las diez mejores cruzas cada una, indicando la excelente aptitud combinatoria general de estas líneas. Entre las líneas reconstituídas, Ac.7843-16S5-F3 y Ac. 7929-38S4-F3 se incluyeron como progenitores en cuatro y tres híbridos superiores, respectivamente. Estas dos líneas reconstituídas de generaciones tempranas parecen tener buena aptitud combinatoria general. El valor de $b_{i}$ para el híbrido con mayor rendimiento, SC07 fué alto (1.35), indicando que está mejor adaptado a ambientes con alto rendimiento, mientras que $\mathrm{SC} 03\left(\mathrm{~b}_{\mathrm{i}}=0.73\right)$ se comporta bien en ambientes de bajo rendimiento. En los dos híbridos, el valor de $\mathrm{SD}_{\mathrm{i} 2}$ fue significativamente diferente de 0 , indicando su falta de estabilidad de rendimiento a través de ambientes. Los híbridos SC11, SCl2, SC06 y SC05 son los más estables de este ensayo (Cuadro 3).
En el ensayo SC9008, el híbrido SC57 fue el más rendidor $(5.95 \mathrm{t} / \mathrm{ha})$ y también fue muy estable a través de localidades $\left(b_{i}=1.04\right)$. Otros híbridos que mostraron estabilidad en este ensayo fueron SC44 y SC30. Las cruzas SC58 y SC36 se desarrollaron bien en localidades de bajo rendimiento, mientras que SC45 y SC48 se adaptan mejor en localidades de alto rendimiento. Al igual que en el ensayo SC9007, las dos líneas reconstituídas de Ac.7843 y Ac.7929 tuvieron buena aptitud combinatoria ya que están incluídos en siete de las diez mejores cruzas.

En el ensayo SC9009, los híbridos SC82, SC80, SC77, SC76 y SC74 se consideran ideales para ambientes de alto rendimiento, mientras que SC61, SC65 y SC62 tuvieron un buen comportamiento en ambientes

Cuadro 3. Medias de rendimiento y parametros de estabilidad (Eberhart y Russell,1966) de 10 cruzas simples superiores de SC 9007, SC 9008, y SC 9009.

\begin{tabular}{|c|c|c|c|c|}
\hline $\begin{array}{l}\text { Código de } \\
\text { Híbridos }\end{array}$ & Genealogía & \multirow[t]{2}{*}{$\begin{array}{l}\text { Rendim. } \\
\text { (t/ha) }\end{array}$} & \multirow[t]{2}{*}{$\frac{\text { Param. }}{\text { bi }}$} & \multirow[t]{2}{*}{$\frac{\text { de estabil. }}{\text { Sdi2 }}$} \\
\hline sc 9007( & (7 Localidades) & & & \\
\hline SC 07 & Pob.21 C5 HC109-S7 x AC.7843-16-S5-F3 & 6,49 & 1,35 & 0,75 \\
\hline sc 11 & Pob.21 C5 HC219-S7 x Ac.7929-38-S4-F3 & 6,32 & 0,92 & 0,06 \\
\hline sc 12 & Pob.21 C5 HC219-S7 x Ac.7843-16-S5-F3 & 6,32 & 1,06 & 0,05 \\
\hline SC 03 & Pob.21 C5 HC229-S7 x AC.7843-16-S5-F3 & 6,09 & 0,73 & 0,46 \\
\hline SC 06 & Pob. 21 C5 HC109-S7 x Ac.7929-38-S4-F3 & 6,01 & 1,03 & $-0,01$ \\
\hline SC 19 & Pob.21 C5 HC84-S5 x Ac.7843-16-S5-F3 & 6,00 & 0,89 & 0,43 \\
\hline SC 09 & Pob.21 C5 HC219-S7 x Pob.22 TSR(S2)-S5-F3 & 5,94 & 1,27 & 0,39 \\
\hline SC 05 & Pob.21 C5 HC109-S7 x Pob.25 CO HC31-S6-F3 & 5,91 & 1,02 & 0,07 \\
\hline SC 15 & Pob.21 C5 HC219-S7 x Ac.7929-38-S4-F3 & 5,82 & 0,94 & 0,10 \\
\hline $\begin{array}{l}\text { SC } 04 \\
\text { Promedio }\end{array}$ & Pob.21 C5 HC109-S7 x Pob.22 TSR(S2)-S5-F3 & $\begin{array}{l}5,81 \\
5,33\end{array}$ & 0,78 & 0,15 \\
\hline \multicolumn{5}{|c|}{ SC 9008 (7 localidades) } \\
\hline SC 57 & P.24 C20 MH94-S5 X AC. 7929-38-S4-F3 & 5,95 & 1,04 & 0,11 \\
\hline SC 48 & P.23 C20 MH268-S5 X Ac.7929-38-S4-F3 & 5,62 & 1,27 & 0,78 \\
\hline SC 44 & Ac. 7643-15-S6 X Pob.32 C6 (S1) MH88-S2-F3 & 5,49 & 1,06 & $-0,10$ \\
\hline SC 45 & Ac. 7643-15-S6 X Pob.22 TSR (S2)-S5-F3 & 5,32 & 1,31 & 0,67 \\
\hline SC 38 & Pob.25 CO HC31-S6 X Ac.7929-38-S4-F3 & 5,19 & 1,25 & 0,27 \\
\hline SC 30 & Pob.25 CO HC31-S6 X Ac.7843-16-S5-F3 & 5,17 & 1,21 & $-0,04$ \\
\hline SC 58 & P.24 C20 MH94-S5 X AC.7843-16-S5-F3 & 5,16 & 0,18 & 0,59 \\
\hline SC 36 & Pob.25 CO HC112-S6 X Ac.7843-16-S5-F3 & 5,11 & 0,66 & 0,29 \\
\hline SC 35 & Pob. 25 CO HC112-S6 X Ac.7929-38-S4-F3 & 4,89 & 1,21 & 0,37 \\
\hline SC 50 & P.24 TSR-24-S4 x Pob.22 TSR (S2)-S5-F3 & 4,84 & 0,94 & 0,33 \\
\hline Promedio & & 4,66 & & \\
\hline \multicolumn{5}{|c|}{ SC 9009 (8 Localidades) } \\
\hline SC 82 & Pob.27 C5 HC117-S4 x Sta. Rosa 8079-S3 & 5,94 & 1,63 & $-0,14$ \\
\hline SC 80 & Pob.24 C5 HC34-S3 x Sta. Rosa 8079-S3 & 5,93 & 1,82 & 0,58 \\
\hline SC 77 & Pob.24 C5 HC34-S3 x Pob.27 C5 HC71-S4 & 5,88 & 1,53 & $-0,03$ \\
\hline SC 61 & Pob.24 C5 HC227-S6 x Pob.27 C5 HC15-S4-F3 & 5,69 & 0,44 & 0,07 \\
\hline SC 65 & Pob. 27 C5 HC217-S5 x Pob.27 C5 HC15-S4-F3 & 5,55 & 0,65 & 0,15 \\
\hline SC 76 & Pob.24 C5 HC34-S3 x Pob.27 C5 HC71-S3 & 5,53 & 1,30 & 0,53 \\
\hline SC 62 & Pob.24 C5 HC227-S5 x Pob.27 C5 HC15-S4-F3 & 5,50 & 0,69 & 0,88 \\
\hline SC 74 & P.26 C19 MH17-S5 x Pob.27 C5 HC15-S4-F3 & 5,40 & 1,65 & 0,44 \\
\hline SC 67 & Pob.28 TSR(S2)-S5 x Pob.27 C5 HC15-S4-F3 & 5,35 & 1,05 & $-0,06$ \\
\hline $\begin{array}{l}\text { SC } 83 \\
\text { Promedio }\end{array}$ & Pob.27 C5 HC117-S4 x AC.8078-S3 & $\begin{array}{l}5,34 \\
5,08\end{array}$ & 1,55 & $-0,04$ \\
\hline
\end{tabular}


desfavorables. SC67 fue el híbridos más estable. Cabe hacer notar que SC82 y SC80 son híbridos convencionales involucrando líneas de generaciones tempranas. En ambos híbridos, uno de los padres (Sta. Rosa 8079-S3) es una línea derivada de la Población 79 que es resistente al achaparramiento. Una línea reconstituída derivada de la familia Pob. 27 HC15 fué progenitor en cinco de las mejores 10 cruzas y parece ser buen combinador (Cuadro 3).

Algunas de las mejores cruzas en el ensayo SC 9007, se comparan con sus respectivos testigos en cada localidad (Cuadro 4).

El más alto rendimiento (39-45\%) sobre los testigos, se observó en Cuyuta, Guatemala. En Omonita, Honduras; los híbridos mostraron una ventaja mínima sobre los testigos; el híbrido SC07 que ocupó el primer lugar en rendimiento, estuvo a la par con el híbrido testigo H-29. La cruza SC07 (Pob. 21 x Pob.43) estuvo en los primeros tres lugares en las cuatro localidades de Centroamérica. SC12, una cruza similar que incluye líneas de Pob. 21 y 43 mostró alto rendimiento en Cuyuta, Guatemala y Omonita, Honduras. En Palmira y Turipaná, Colombia; Poza Rica, México; el híbrido SC11 (Pob. 21 $x$ Pob. 29) estuvo entre los tres primeros lugares. La cruza SC03 rindió bién en Colombia, ocupando el primer y segundo lugar en Palmira y Turipaná respectivamente.

Las mejores cruzas del ensayo SC 9008 se presentan en el Cuadro 5 por localidades individuales.

Se observaron las ganancias más altas de híbridos sobre testigos en las dos localidades de Guatemala.

Cuadro 4. Cruzas con alto rendimiento de SC 9007 en diferentes localidades.

\begin{tabular}{|c|c|c|c|c|}
\hline $\begin{array}{l}\text { Localidad } \\
\text { y País }\end{array}$ & $\begin{array}{l}\text { Código de } \\
\text { Híbridos }\end{array}$ & Genealogía & $\begin{array}{l}\text { Rend. } \\
\text { (t/ha) }\end{array}$ & $\begin{array}{c}\% \text { sobre } \\
\text { mejor test. }\end{array}$ \\
\hline $\begin{array}{l}\text { Cuyuta, } \\
\text { Guatemala }\end{array}$ & $\begin{array}{l}\text { SC } 12 \\
\text { SC } 13 \\
\text { SC } 07 \\
\text { Testigo } 1 \\
\text { Testigo } 2\end{array}$ & $\begin{array}{l}\text { Pob.21 C5 HC219-S7 } \times \text { Ac. } 7843-16-S 5-F 3 \\
\text { Pob.21 C5 HC219-S7 } \times \text { Pob.22 TSR(S2)-S5-F3 } \\
\text { Pob.21 C5 HC109-S7 } \times \text { AC. } 7843-16-S 5-F 3 \\
\text { GB35 } \times \text { GB41 } \\
\text { GB43 } \times \text { GB45 }\end{array}$ & $\begin{array}{l}5,94 \\
5,78 \\
5,70 \\
4,11 \\
3,74\end{array}$ & $\begin{array}{l}144,5 \\
140,6 \\
138,7\end{array}$ \\
\hline $\begin{array}{l}\text { San Andrés, } \\
\text { EL Salvador }\end{array}$ & $\begin{array}{l}\text { SC } 07 \\
\text { SC } 04 \\
\text { Testigo } 1\end{array}$ & $\begin{array}{l}\text { Pob.21 C5 HC109-S7 } \times \text { AC. } 7843-16-S 5-F 3 \\
\text { Pob.21 C5 HC109-S7 } \times \text { Pob. } 22 \text { TSR(S2)-S5-F3 } \\
\text { S28 } \times 6-283\end{array}$ & $\begin{array}{l}6,20 \\
5,66 \\
5,48\end{array}$ & $\begin{array}{l}113,1 \\
103,3\end{array}$ \\
\hline $\begin{array}{l}\text { Omonita, } \\
\text { Honduras }\end{array}$ & $\begin{array}{l}\text { SC } 07 \\
\text { SC } 12 \\
\text { Testigo } 1 \\
\text { Testigo } 2\end{array}$ & $\begin{array}{l}\text { Pob. } 21 \text { C5 HC109-S7 x AC. } 7843-16-S 5-F 3 \\
\text { Pob.21 C5 HC219-S7 x AC. } 7843-16-S 5-F 3 \\
\text { H } 27 \\
\text { H } 29\end{array}$ & $\begin{array}{l}9,39 \\
8,17 \\
8,84 \\
9,23\end{array}$ & $\begin{array}{r}101,7 \\
88,5\end{array}$ \\
\hline $\begin{array}{l}\text { EEEJN, } \\
\text { Costa Rica }\end{array}$ & $\begin{array}{l}\text { SC } 26 \\
\text { SC } 15 \\
\text { SC } 07 \\
\text { Testigo } 1 \\
\text { Testigo } 2\end{array}$ & $\begin{array}{l}\text { Pob.22 TSR(S2)-S5 x AC.7843-16-S5-F3 } \\
\text { Pob.21 C5 HC219-S7 x AC.7929-38-S4-F3 } \\
\text { Pob. } 21 \text { C5 HC109-S7 x AC.7843-16-S5-F3 } \\
\text { EJN } 2 \\
\text { Los Diamantes } 8043\end{array}$ & $\begin{array}{l}4,83 \\
4,70 \\
4,60 \\
3,80 \\
2,57\end{array}$ & $\begin{array}{l}127,1 \\
123,7 \\
121,1\end{array}$ \\
\hline $\begin{array}{l}\text { Palmira, } \\
\text { Colombia }\end{array}$ & $\begin{array}{l}\text { SC } 03 \\
\text { SC } 12 \\
\text { SC } 11 \\
\text { Testigo } 1 \\
\text { Testigo } 2\end{array}$ & 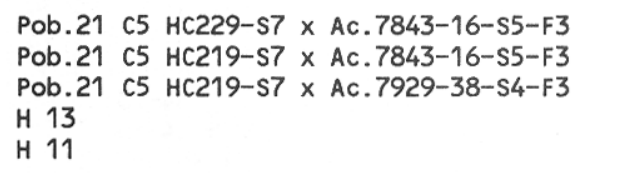 & $\begin{array}{l}6,61 \\
6,58 \\
5,84 \\
5,49 \\
3,20\end{array}$ & $\begin{array}{l}120,4 \\
119,9 \\
106,4\end{array}$ \\
\hline $\begin{array}{l}\text { Turipana, } \\
\text { Colombia }\end{array}$ & $\begin{array}{l}\text { SC } 19 \\
\text { SC } 03 \\
\text { SC } 11 \\
\text { Testigo } 1 \\
\text { Testigo } 2\end{array}$ & $\begin{array}{l}\text { Pob. } 21 \text { C5 HC84-S5 x AC. } 7843-16-S 5-F 3 \\
\text { Pob.21 C5 HC229-S7 X AC.7843-16-S5-F3 } \\
\text { Pob.21 C5 HC219-S7 X AC.7929-38-S4-F3 } \\
\text { HE } 1023 \\
\text { ICA V1560 }\end{array}$ & $\begin{array}{l}7,36 \\
7,20 \\
7,06 \\
5,47 \\
4,96\end{array}$ & $\begin{array}{l}134,6 \\
131,6 \\
129,1\end{array}$ \\
\hline $\begin{array}{l}\text { Poza Rica, } \\
\text { México }\end{array}$ & $\begin{array}{l}\text { SC } 09 \\
\text { SC } 11 \\
\text { SC } 15 \\
\text { Testigo } 1 \\
\text { Testigo } 2\end{array}$ & $\begin{array}{l}\text { Pob.21 C5 HC219-S7 x Pob.22 TSR(S2)-S5-F3 } \\
\text { Pob.21 C5 HC219-S7 x AC.7929-38-S4-F3 } \\
\text { Pob.21 C5 HC219-S7 x AC.7929-38-S4-F3 } \\
\text { B-833 } \\
\text { SC (Pool } 24 \times \text { Pob.21) }\end{array}$ & $\begin{array}{l}7,35 \\
7,02 \\
6,73 \\
5,31 \\
4,84\end{array}$ & $\begin{array}{l}138,4 \\
132,2 \\
126,7\end{array}$ \\
\hline
\end{tabular}


Cuadro 5. Cruzas con alto rendimiento de SC 9008 en diferentes localidades.

\begin{tabular}{|c|c|c|c|c|}
\hline $\begin{array}{l}\text { Localidad } \\
\text { y País }\end{array}$ & $\begin{array}{l}\text { Código de } \\
\text { Híbridos }\end{array}$ & Genealogía & $\begin{array}{l}\text { Rend. } \\
(t / h a)\end{array}$ & $\begin{array}{c}\% \text { sobre } \\
\text { mejor test. }\end{array}$ \\
\hline $\begin{array}{l}\text { Cuyuta, } \\
\text { Guatemala }\end{array}$ & $\begin{array}{l}\text { SC } 57 \\
\text { SC } 48 \\
\text { SC } 38 \\
\text { Testigo } 1 \\
\text { Testigo } 2\end{array}$ & $\begin{array}{l}\text { P.24 C20 MH94-S5 X AC. } 7929-38-S 4-F 3 \\
\text { P.23 C20 MH268-S5 X AC.7929-38-S4-F3 } \\
\text { Pob.25 CO HC31-S6 X AC.7929-38-S4-F3 } \\
\text { GB35 x GB41 } \\
\text { GB43 x GB45 }\end{array}$ & $\begin{array}{l}6,10 \\
5,89 \\
5,85 \\
4,56 \\
4,55\end{array}$ & $\begin{array}{l}133,8 \\
129,2 \\
128,3\end{array}$ \\
\hline $\begin{array}{l}\text { Las Vegas, } \\
\text { Guatemala }\end{array}$ & $\begin{array}{l}\text { SC } 48 \\
\text { SC } 58 \\
\text { SC } 56 \\
\text { Testigo } 1 \\
\text { Testigo } 2\end{array}$ & $\begin{array}{l}\text { P.23 C20 MH268-S5 X AC.7929-38-S4-F3 } \\
\text { P.24 C20 MH94-S5 X Ac. } 7843-16-S 5-F 3 \\
\text { P.24 C20 MH94-S5 X Pob.25 CO HC31-S6-F3 } \\
\text { Testigo local } 1 \\
\text { Testigo local } 2\end{array}$ & $\begin{array}{l}5,32 \\
5,00 \\
4,54 \\
3,67 \\
3,63\end{array}$ & $\begin{array}{l}145,0 \\
136,2 \\
123,7\end{array}$ \\
\hline $\begin{array}{l}\text { San Andrés, } \\
\text { El Salvador }\end{array}$ & $\begin{array}{l}\text { SC } 51 \\
\text { SC } 57 \\
\text { SC } 38 \\
\text { Testigo } 1\end{array}$ & $\begin{array}{l}\text { P. } 24 \text { TSR-24-S4 X Pob. } 25 \text { CO HC31-S6-F3 } \\
\text { P.24 C20 MH94-S5 X AC. } 7929-38-S 4-F 3 \\
\text { Pob. } 25 \text { CO HC31-S6 X AC.7929-38-S4-F3 } \\
\text { S28 x 6-283 4,90 }\end{array}$ & $\begin{array}{l}5,18 \\
5,08 \\
5,05\end{array}$ & $\begin{array}{l}105,7 \\
103,7 \\
103,1\end{array}$ \\
\hline $\begin{array}{l}\text { EEEJN, } \\
\text { Costa Rica }\end{array}$ & $\begin{array}{l}\text { SC } 57 \\
\text { SC } 36 \\
\text { SC } 58 \\
\text { Testigo } 1 \\
\text { Testigo } 2\end{array}$ & $\begin{array}{l}\text { P. } 24 \text { C20 MH94-S5 X AC. } 7929-38-S 4-F 3 \\
\text { Pob. } 25 \text { CO HC112-S6 X AC. } 7843-16-S 5-F 3 \\
\text { P. } 24 \text { C20 MH94-S5 X AC. } 7843-16-S 5-F 3 \\
\text { Los Diamantes } 8043 \\
\text { EJN } 2\end{array}$ & $\begin{array}{l}6,29 \\
5,93 \\
5,52 \\
2,35 \\
4,02\end{array}$ & $\begin{array}{l}156,5 \\
147,5 \\
137,3\end{array}$ \\
\hline $\begin{array}{l}\text { Palmira, } \\
\text { Colombia }\end{array}$ & $\begin{array}{l}\text { SC } 48 \\
\text { SC } 45 \\
\text { SC } 57 \\
\text { Testigo } 1 \\
\text { Testigo } 2\end{array}$ & $\begin{array}{l}\text { P. } 23 \text { C20 MH268-S5 X Ac.7929-38-S4-F3 } \\
\text { AC. } 7643-15-S 6 \text { X Pob. } 22 \text { TSR (S2)-S5-F3 } \\
\text { P. } 24 \text { C20 MH94-S5 X AC. } 7929-38-S 4-F 3 \\
\text { H } 13 \\
\text { H } 12\end{array}$ & $\begin{array}{l}8,45 \\
7,36 \\
7,31 \\
6,07 \\
7,64\end{array}$ & $\begin{array}{r}110,6 \\
96,3 \\
95,7\end{array}$ \\
\hline $\begin{array}{l}\text { Turipana, } \\
\text { Colombia }\end{array}$ & $\begin{array}{l}\text { SC } 57 \\
\text { SC } 58 \\
\text { SC } 49 \\
\text { Testigo } 1 \\
\text { Testigo } 2\end{array}$ & $\begin{array}{l}\text { P.24 C20 MH94-S5 X AC.7929-38-S4-F3 } \\
\text { P.24 C20 MH94-S5 X AC. } 7843-16-S 5-F 3 \\
\text { P.23 C20 MH268-S5 X AC.7843-16-S5-F3 } \\
\text { ICA V1560 } \\
\text { HE } 1023\end{array}$ & $\begin{array}{l}6,66 \\
6,34 \\
6,16 \\
5,61 \\
5,31\end{array}$ & $\begin{array}{l}118,7 \\
113,0 \\
109,8\end{array}$ \\
\hline $\begin{array}{l}\text { Poza Rica, } \\
\text { México }\end{array}$ & $\begin{array}{l}\text { SC } 45 \\
\text { SC } 33 \\
\text { SC } 44 \\
\text { Testigo } 1 \\
\text { Testigo } 2\end{array}$ & $\begin{array}{l}\text { Ac. } 7643-15-\mathrm{S} 6 \times \text { X Pob. } 22 \text { TSR (S2)-S5-F3 } \\
\text { Pob. } 25 \text { CO HC112-S6 X Pob.32 C6(S1) MH88-S2 } \\
\text { Ac. } 7643-15-S 6 \text { X Pob. } 32 \text { C6 (S1) MH88-S2-F3 } \\
\text { B-833 } \\
\text { SC (Pool } 24 \times \text { Pob.21) }\end{array}$ & $\begin{array}{l}6,79 \\
6,12 \\
6,00 \\
6,02 \\
5,18\end{array}$ & $\begin{array}{r}112,8 \\
101,7 \\
99,7\end{array}$ \\
\hline
\end{tabular}

Las menores ganancias sobre los testigos se observaron en San Andrés, El Salvador y Palmira, Colombia. La cruza SC57, que incluye líneas del Pool 24 y la Pob. 29, ocupó los tres lugares en rendimiento en cinco de siete localidades por lo que se considera el híbrido más estable y de más alto rendimiento. El híbrido SC38, una cruza de grano cristalino $\mathrm{x}$ dentado, se comportó bien en localidades de Centro América. La cruza SC 48, otra combinación de grano cristalino x dentado (Pool $23 \mathrm{x}$ Pob. 29), mostró un alto rendimiento en Cuyuta, Las Vegas, Guatemala; Palmira, Colombia.

En el Cuadro 6, se presentan para cada una de las ocho localidades, los híbridos amarillos de alto rendimiento que se incluyeron en el ensayo SC 9009. Las mayores ganancias sobre los testigos se observaron en Cuyuta, Guatemala; Santa Cruz, El Salvador. En Las Acacias, Honduras; se observaron ganancias mínimas sobre los testigos. El híbrido SC61 (Pob. 24 x Pob.27) fué uno de los más rendidores en Guatemala, El Salvador y Honduras. SC65, un híbrido intrapoblacional entre líneas de la Pob. 27, se comportó bien en Guatemala y El Salvador. Los híbridos SC77, SC82 y SC80 fueron los de más alto rendimiento en ambas localidades de Panamá, mientras que SC80 fue el de más alto rendimiento en Turipaná, Colombia; Poza Rica, México. 
Cuadro 6. Cruzas con alto rendimiento de SC 9009 en diferentes localidades.

\begin{tabular}{|c|c|c|c|c|}
\hline $\begin{array}{l}\text { Localidad } \\
\text { y País }\end{array}$ & $\begin{array}{l}\text { Código de } \\
\text { Híbridos }\end{array}$ & Genealogía & $\begin{array}{l}\text { Rend. } \\
\text { (t/ha) }\end{array}$ & $\begin{array}{c}\% \text { sobre } \\
\text { mejor test. }\end{array}$ \\
\hline $\begin{array}{l}\text { Cuyuta, } \\
\text { Guatemala }\end{array}$ & $\begin{array}{l}\text { SC } 74 \\
\text { SC } 61 \\
\text { SC } 65 \\
\text { Testigo } 1 \\
\text { Testigo } 2\end{array}$ & $\begin{array}{l}\text { P.26 C19 MH17-S5 x Pob.27 C5 HC15-S4-F3 } \\
\text { Pob.24 C5 HC227-S6 x Pob.27 C5 HC15-S4-F3 } \\
\text { Pob.27 C5 HC217-S5 x Pob.27 C5 HC15-S4-F3 } \\
\text { HA } 46 \\
\text { A } 6\end{array}$ & $\begin{array}{l}5,86 \\
5,37 \\
5,35 \\
4,30 \\
3,69\end{array}$ & $\begin{array}{l}136,3 \\
124,9 \\
124,4\end{array}$ \\
\hline $\begin{array}{l}\text { Santa Cruz, } \\
\text { EL Salvador }\end{array}$ & $\begin{array}{l}\text { SC } 62 \\
\text { SC } 61 \\
\text { SC } 65 \\
\text { Testigo } 1 \\
\text { Testigo } 2\end{array}$ & 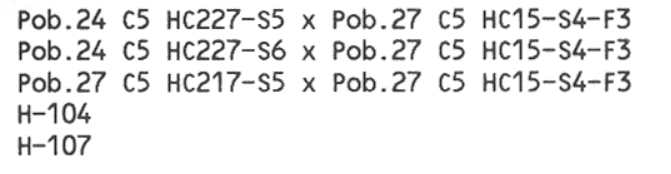 & $\begin{array}{l}6,26 \\
5,75 \\
5,54 \\
4,53 \\
3,24\end{array}$ & $\begin{array}{l}138,2 \\
126,9 \\
122,3\end{array}$ \\
\hline $\begin{array}{l}\text { Las Acacias, } \\
\text { Honduras }\end{array}$ & $\begin{array}{l}\text { SC } 61 \\
\text { SC } 69 \\
\text { Testigo } 1 \\
\text { Testigo } 2\end{array}$ & $\begin{array}{l}\text { Pob.24 C5 HC227-S6 x Pob.27 C5 HC15-S4-F3 } \\
\text { Pob.28 TSR(S2)-21-S4 x Pob.27 C5 HC15-S4-F3 } \\
\text { H-29 } \\
\text { B-102 }\end{array}$ & $\begin{array}{l}5,53 \\
5,43 \\
5,47 \\
4,03\end{array}$ & $\begin{array}{r}101,1 \\
99,3\end{array}$ \\
\hline $\begin{array}{l}\text { La Honda, } \\
\text { Panamá }\end{array}$ & $\begin{array}{l}\text { SC } 77 \\
\text { SC } 82 \\
\text { SC } 80 \\
\text { Testigo } 1 \\
\text { Testigo } 2\end{array}$ & $\begin{array}{l}\text { Pob.24 C5 HC34-S3 x Pob.27 C5 HC71-S4 } \\
\text { Pob.27 C5 HC117-S4 x Sta. Rosa 8079-S3 } \\
\text { Pob.24 C5 HC34-S3 x Sta. Rosa 8079-S3 } \\
\text { X-304 C } \\
\text { X-3214 }\end{array}$ & $\begin{array}{l}7,37 \\
7,13 \\
7,08 \\
5,98 \\
6,29\end{array}$ & $\begin{array}{l}117,2 \\
113,4 \\
112,6\end{array}$ \\
\hline $\begin{array}{l}\text { Parita, } \\
\text { Panamá }\end{array}$ & $\begin{array}{l}\text { SC } 80 \\
\text { SC } 77 \\
\text { SC } 82 \\
\text { Testigo } 1 \\
\text { Testigo } 2\end{array}$ & $\begin{array}{l}\text { Pob.24 C5 HC34-S3 x Sta. Rosa 8079-S3 } \\
\text { Pob.24 C5 HC34-S3 x Pob.27 C5 HC71-S4 } \\
\text { Pob.27 C5 HC117-S4 x Sta. Rosa 8079-S3 } \\
\text { Testigo local-1 } \\
\text { Testigo local-2 }\end{array}$ & $\begin{array}{l}8,05 \\
7,08 \\
6,82 \\
5,88 \\
6,43\end{array}$ & $\begin{array}{l}125,2 \\
110,1 \\
106,1\end{array}$ \\
\hline $\begin{array}{l}\text { Palvira, } \\
\text { Colombia }\end{array}$ & $\begin{array}{l}\text { SC } 82 \\
\text { SC } 74 \\
\text { SC } 83 \\
\text { Testigo } 1 \\
\text { Testigo } 2\end{array}$ & $\begin{array}{l}\text { Pob.27 C5 HC117-S4 x Sta. Rosa 8079-S3 } \\
\text { P. } 26 \text { C19 MH17-S5 x Pob. } 27 \text { C5 HC15-S4-F3 } \\
\text { Pob. } 27 \text { C5 HC117-S4 x AC. } 8078-S 3 \\
\text { CIMCALI } 11 \\
\text { CIMCALI } 12\end{array}$ & $\begin{array}{l}7,83 \\
7,67 \\
7,52 \\
6,65 \\
6,96\end{array}$ & $\begin{array}{l}112,5 \\
110,2 \\
108,0\end{array}$ \\
\hline $\begin{array}{l}\text { Turipana, } \\
\text { Colombia }\end{array}$ & $\begin{array}{l}\text { SC } 76 \\
\text { SC } 78 \\
\text { SC } 80 \\
\text { Testigo } 1 \\
\text { Testigo } 2\end{array}$ & $\begin{array}{l}\text { Pob. } 24 \text { C5 HC34-S3 x Pob. } 27 \text { C5 HC71-S3 } \\
\text { Pob. } 24 \text { C5 HC34-S3 x Pob.27 C5 HC117-S3 } \\
\text { Pob.24 C5 HC34-S3 x Sta. Rosa } 8079-S 3 \\
\text { ICA V156 } \\
\text { HE } 1023\end{array}$ & $\begin{array}{l}5,65 \\
5,65 \\
5,50 \\
4,65 \\
3,73\end{array}$ & $\begin{array}{l}121,5 \\
121,5 \\
118,3\end{array}$ \\
\hline $\begin{array}{l}\text { Poza Rica, } \\
\text { México }\end{array}$ & $\begin{array}{l}\text { SC } 80 \\
\text { SC } 62 \\
\text { Testigo } 1 \\
\text { Testigo } 2\end{array}$ & 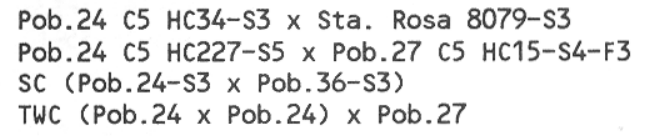 & $\begin{array}{l}6,70 \\
6,29 \\
6,17 \\
6,09\end{array}$ & $\begin{array}{l}108,6 \\
101,9\end{array}$ \\
\hline
\end{tabular}

En muchas de las localidades, los testigos fueron los mejores híbridos comerciales, por lo que el comportamiento superior de híbridos simples experimentales de CIMMYT en muchas de las localidades de Centro y Sudamérica, es prometedor.

\section{CONCLUSIONES}

1) Los resultados de SC 9007 y SC 9008, mostraron que estos híbridos blancos tienen potencial en Guatemala, Costa Rica, El Salvador y Colombia. Los híbridos amarillos en el ensayo SC 9009, se comportaron bien en
Guatemala, El Salvador, Panamá y Colombia; comparados con los híbridos testigo.

2) Entre los híbridos blancos, SC11, SCl2, SC57 y SC44 son los más estables a través de ambientes. La cruza SC67 fue la más estable entre los híbridos amarillos; aunque en rendimiento ocupó sólo el noveno lugar entre 23 híbridos.

3) Las dos localidades de Colombia y Poza Rica, México; favorecieron grupos diferentes de híbridos, en comparación con otras localidades de Centroamérica. 
4) Los híbridos blancos SC07, SC12, SC38, SC48, SC57 y los dos híbridos amarillos SC61 y SC65 se comportaron bien en localidades de Centroamérica. Sin embargo, en Panamá se comportaron mejor los híbridos SC77, SC80 y SC82. Entre los híbridos blancos SC57, $\mathrm{SC0} 3$ y SCU se consideran como las mejores opciones para Colombia.

\section{BIBLIOGRAFIA}

BECK, D.L., S.K. VASAL, AND J. CROSSA. 1990. Heterosis and combining ability of CIMMYT 's tropical early and intermediate maturity maize germplasm. Maydica 35(3):279-285.

CROSSA, J., S.K. VASAL, AND D.L. BECK. 1990. Combining ability study in diallel crosses of CIMMYT 's tropical late yellow maize germplasm. Maydica 35(3):273-278.

EBERHART, SA., AND W.L. RUSSELL. 1966. Stability parameters for comparing varieties. Crop Sci. 6:36-40.

VASAL, S.K., D.L. BECK, AND J. CROSSA. 1986. Studies on the combining ability of CIMMYT maize germplasm.
CIMMYT Research Highlights 1986. CIMMYT, El Batan, Mexico.

VASAL, S.K., G. HAN, E. ELIAS, AND N. VERGARA. 1988. Desarrollo de híbridos no convencionales de maíz. Presentado en la XIII Reunión de Maiceros de la Zona Andina, Chiclayó, Perú; del 25 al 30 de Septiembre, 1988.

VASAL, S.K., G. HAN, AND N. VERGARA. 1990. Conceptos y resultados de ensayos de híbridos de maíz (Zea mays L.) no convencionales. A paper presented at XIII Congreso Nacional de Fitogenética. Cd. Juárez, México. 3-7 de Septiembre, 1990. p.282.

VASAL, S.K., AND G. SRINIVASAN. 1991. Breeding strategies to meet changing trends in hybrid maize development. Presented at the Golden Jubilee of the Indian Society of Genetics and Plant Breeding. New Delhi. 12-15 Feb. 1991. New Delhi, India.

VASAL, S.K., G. SRINIVASAN, D.L. BECK, J. CROSSA, S. PANDEY, AND C. DE LEON. 1992b. Heterosis and combining ability of CIMMYT's tropical late white germplasm. Maydica (In press). 